\title{
Drug retention rates and relevant risk factors for drug discontinuation due to adverse events in rheumatoid arthritis patients receiving anticytokine therapy with different target molecules
}

\author{
Ryoko Sakai, ${ }^{1,2}$ Michi Tanaka, ${ }^{1,2}$ Toshihiro Nanki, ${ }^{1,2}$ Kaori Watanabe ${ }^{1,2}$ Hayato \\ Yamazaki ${ }^{1,2}$ Ryuji Koike, ${ }^{1,2,3}$ Hayato Nagasawa, ${ }^{4}$ Koichi Amano, ${ }^{4}$ Kazuyoshi Saito, ${ }^{5}$ \\ Yoshiya Tanaka, ${ }^{5}$ Satoshi Ito, ${ }^{6}$ Takayuki Sumida, ${ }^{6}$ Atsushi Ihata, ${ }^{7}$ Yoshiaki Ishigatsubo, ${ }^{7}$ \\ Tatsuya Atsumi, ${ }^{8}$ Takao Koike, ${ }^{8}$ Atsuo Nakajima, ${ }^{9}$ Naoto Tamura, ${ }^{10}$ Takao Fujii, ${ }^{11}$ Hiroaki \\ Dobashi, ${ }^{12}$ Shigeto Tohma, ${ }^{13}$ Takahiko Sugihara, ${ }^{14}$ Yukitaka Ueki, ${ }^{15}$ Akira Hashiramoto, ${ }^{16}$ \\ Atsushi Kawakami, ${ }^{17}$ Noboru Hagino, ${ }^{18}$ Nobuyuki Miyasaka, ${ }^{2,19}$ Masayoshi Harigai 1,2,3 \\ for the REAL Study Group
}

\begin{abstract}
- Additional supplementary data are published online only. To view these files please visit the journal online (http://ard.bmj. com/content/early/recent)
\end{abstract}

For numbered affiliations see end of article

\section{Correspondence to \\ Masayoshi Harigai, Department of Pharmacovigilance, Graduate School of Medical and Dental Sciences, Tokyo Medical and Dental University, 1-5-45 Yushima, Bunkyo-ku, Tokyo, 113-8519, Japan; mharigai.mpha@tmd.ac.jp}

Received 29 September 2011 Accepted 25 February 2012 Published Online First 13 April 2011

\begin{abstract}
Objective To compare reasons for discontinuation and drug retention rates per reason among anticytokine therapies, infliximab, etanercept and tocilizumab, and the risk of discontinuation of biological agents due to adverse events (AE) in patients with rheumatoid arthritis (RA).

Method This prospective cohort study included Japanese RA patients who started infliximab ( $n=412,636.0$ patientyears (PY)), etanercept ( $n=442,765.3 \mathrm{PY})$, or tocilizumab ( $n=168,206.5$ PY) as the first biological therapy after their enrolment in the Registry of Japanese Rheumatoid Arthritis Patients for Long-term Safety (REAL) database. Drug retention rates were calculated using the KaplanMeier method. To compare risks of drug discontinuation due to AE for patients treated with these biological agents, the Cox proportional hazard model was applied.

Results The authors found significant differences among the three therapeutic groups in demography, clinical status, comorbidities and usage of concomitant drugs. Development of AE was the most frequent reason for discontinuation of biological agents in the etanercept and tocilizumab groups, and the second most frequent reason in the infliximab group. Discontinuation due to good control was observed most frequently in the infliximab group. Compared with etanercept, the use of infliximab (HR 1.69; 95\% Cl 1.14 to 2.51) and tocilizumab (HR 1.98; 95\% Cl 1.04 to 3.76) was significantly associated with a higher risk of discontinuation of biological agents due to $\mathrm{AE}$.

Conclusions Reasons for discontinuation are significantly different among biological agents. The use of infliximab and tocilizumab was significantly associated with treatment discontinuation due to AE compared with etanercept.
\end{abstract}

Biological disease-modifying antirheumatic drugs (biological agents) are a standard treatment for rheumatoid arthritis (RA). ${ }^{12}$ A number of clinical trials have demonstrated that biological agents significantly improve signs and symptoms of RA patients with both early and established disease, and that remission of RA can be achieved with biological agents not only in early RA patients, but also in established RA patients who have shown inadequate responses to conventional non-biological disease-modifying antirheumatic drugs (DMARD).

In Japan, six biological agents have been approved for the treatment of RA, infliximab in 2002, etanercept in 2005, tocilizumab and adalimumab in 2008, abatacept in 2010 and golimumab in 2011. These drugs are widely used in clinical practice according to treatment guidelines for biological agents by the Japan College of Rheumatology ${ }^{34}$ and Japanese drug package inserts. Postmarketing surveillance and some clinical studies have shown short-term effectiveness and safety of these biological agents for Japanese RA patients. ${ }^{5-8}$ The European League Against Rheumatism recommendations for the management of RA state that a tumour necrosis factor (TNF) antagonist should be administered as the first biological DMARD for patients who fail to respond to non-biological DMARD, including methotrexate, ${ }^{9}$ whereas Japanese guidelines do not clearly specify the precedence of biological agents.

Some RA patients treated with biological agents are compelled to stop the administration of these drugs because of lack of efficacy (LOE), adverse events (AE), or financial reasons. In addition, some RA patients discontinue biological agents in the hope of a biological-free remission or biologicalfree low disease activity status. ${ }^{10-12}$ In general, drugs with high retention rates have a good balance between long-term effectiveness and tolerability, reflecting the satisfaction of patients and doctors with the treatment. Because treatment for RA continues for many years or is life-long in the majority of patients, the examination of long-term drug retention rates using a prospective cohort study is important for the evaluation of biological agents.

To establish better treatment strategies for RA, it is important to identify reasons and risk factors causing the discontinuation of a drug, especially for biological agents. Several studies have shown that 
Table 1 Characteristics of RA patients treated with infliximab, etanercept or tocilizumab at the start of the observation period

\begin{tabular}{|c|c|c|c|c|}
\hline & Infliximab group & Etanercept group & Tocilizumab group & \\
\hline & $(n=412)$ & $(n=442)$ & $(n=168)$ & p Value \\
\hline Age, years & $53.6 \pm 13.5$ & $58.5 \pm 13.0$ & $59.8 \pm 13.4$ & $<0.001$ \\
\hline Female, \% & 85.9 & 78.1 & 80.4 & 0.011 \\
\hline Disease duration, years & $7.9 \pm 7.8$ & $10.3 \pm 8.9$ & $10.3 \pm 9.6$ & $<0.001$ \\
\hline Steinbrocker's class (3 or 4 ), $\%$ & 24.8 & 31.2 & 27.4 & 0.108 \\
\hline Steinbrocker's stage (III or IV), \% & 43.9 & 57.0 & 46.4 & $<0.001$ \\
\hline DAS28 (3/CRP) & $4.5 \pm 1.2(n=411)$ & $4.5 \pm 1.3(n=440)$ & $5.1 \pm 3.4(n=167)$ & 0.056 \\
\hline Use of $\geq 3$ previous non-biological DMARD, $\%$ & 41.0 & 54.5 & 31.5 & $<0.001$ \\
\hline Biological--naive, \% & 96.4 & 83.9 & 46.4 & $<0.001$ \\
\hline Methotrexate use, \% & 99.3 & 44.6 & 44.0 & $<0.001$ \\
\hline Methotrexate dose, mg/week & $8.0 \pm 2.1$ & $7.0 \pm 2.0$ & $8.2 \pm 2.9$ & $<0.001$ \\
\hline Use of immunosuppressive drugs, except for methotrexate, $\%$ & 1.9 & 5.7 & 14.9 & $<0.001$ \\
\hline Oral corticosteroid use, $\%$ & 68.9 & 73.1 & 60.1 & 0.008 \\
\hline Prednisolone-equivalent dose of corticosteroids (mg/day) & $5.4 \pm 2.6$ & $6.1 \pm 3.3$ & $4.9 \pm 2.2$ & $<0.001$ \\
\hline Chronic pulmonary disease, $\%$ & 22.6 & 36.7 & 40.5 & $<0.001$ \\
\hline Diabetes mellitus, $\%$ & 8.5 & 14.9 & 12.5 & 0.015 \\
\hline
\end{tabular}

CRP, C-reactive protein; DAS28, disease activity score including 28-joint count; DMARD, disease-modifying antirheumatic drug; RA, rheumatoid arthritis

a frequent reason for the discontinuation of biological agents is the development of AE. ${ }^{5-7} 13-16 \mathrm{Mid}$ to long-term tolerability of TNF inhibitors ${ }^{6131416-24}$ and tocilizumab ${ }^{71525}$ has been reported, and some studies have directly compared drug retention rates among TNF inhibitors or between TNF inhibitors and other biological agents. ${ }^{14} 1617$ 25-27 To summarise, infliximab had the lowest overall retention rate among infliximab, etanercept and adalimumab ${ }^{141617}$ and among infliximab, etanercept and anakinra. ${ }^{26}$ A recent report from the CORRONA registry demonstrated the highest retention rate of infliximab compared with etanercept and adalimumab. ${ }^{27}$ However, drug retention rates have not been compared between TNF inhibitors and the interleukin- 6 receptor inhibitor, tocilizumab, in the real world. In addition, the risk factors causing drug discontinuation due to $\mathrm{AE}$ for patients given these biological agents have not been thoroughly evaluated.

The purpose of this study was to compare drug retention rates and reasons for discontinuation of infliximab, etanercept and tocilizumab among Japanese RA patients, and to investigate the association of the use of these biological agents and other clinical characteristics with drug discontinuation due to AE.

\section{PATIENTS AND METHODS}

\section{Database}

The Registry of Japanese Rheumatoid Arthritis Patients for Longterm Safety (REAL) is an ongoing prospective cohort established to investigate the long-term safety of biological agents in RA patients. Twenty-seven institutions participate, including 16 university hospitals and 11 referring hospitals. Details of REAL have previously been described. ${ }^{28} 29$ Briefly, the criteria for enrolment in REAL include patients meeting the 1987 American College of Rheumatology criteria for RA, written informed consent, and starting or switching treatment with biological agents or starting, adding or switching non-biological DMARD at the time of enrolment in the study. Enrolment in the REAL database was started in June 2005 and closed in January 2012. To facilitate enrolment to the REAL registry, participating physicians were asked to enrol their patients already registered in postmarketing surveillance programmes previously implemented by pharmaceutical companies for biological agents. ${ }^{58}$ In addition, our investigators were also encouraged to enrol as many patients as possible who fulfilled the inclusion criteria. ${ }^{29}$
Data were retrieved from the REAL database on 4 April 2011 for this study. The REAL study was approved by the ethics committees of the Tokyo Medical and Dental University Hospital and other participating institutions.

\section{Data collection}

Each patient's recorded baseline dataincluded demography, disease activity, physical disability, comorbidities, treatments and laboratory data at the beginning of the observation period. A follow-up form was submitted by the site investigators every 6 months to the REAL data centre at the Department of Pharmacovigilance of Tokyo Medical and Dental University to report the occurrence of serious AE, current RA disease activity, treatments and clinical laboratory data. ${ }^{28} 29$ We collected the Steinbrocker class ${ }^{30}$ as the baseline measurement for each patient's physical disability, instead of the health assessment questionnaire disability index. ${ }^{31}$ The investigators in each hospital confirmed the accuracy of their data submitted to the REAL data centre. The centre examined all the data sent by site investigators and sent queries if necessary to verify the accuracy of the data.

\section{Patients}

By April 2011, 2067 RA patients were registered in REAL, of these 1044 patients started treatment with infliximab, etanercept or tocilizumab at the time of enrolment or after enrolment in REAL. Four patients were excluded from this study because the reason for discontinuation of the initial biological agents was not identified. Eighteen patients who were enrolled in another clinical study requiring the discontinuation of infliximab were also excluded. We did not include patients who used adalimumab, abatacept or golimumab as the first biological agent in REAL because we did not have sufficient numbers of patients on adalimumab in the database $(n=98)$ compared with infliximab and etanercept and had no patients given abatacept or golimumab in the database at the time our data were compiled. Our analysis included 412 patients who started infliximab, 442 patients who started etanercept and 168 patients who started tocilizumab.

\section{Follow-up}

For patients who initiated biological agents (infliximab, etanercept, or tocilizumab) at enrolment in REAL, the start date 
Table 2 Reasons for drug discontinuation in RA patients treated with infliximab, etanercept or tocilizumab*

\begin{tabular}{|c|c|c|c|}
\hline Reason for discontinuation & Infliximab $(n=157) \dagger$ & Etanercept $(n=130) \dagger$ & Tocilizumab $(n=51) \dagger$ \\
\hline Adverse events & 57 Cases $(36.3 \%)$ & 57 Cases $(43.8 \%)$ & 23 Cases (45.1\%) \\
\hline Infection & 20 Cases $(12.7 \%)$ & 22 Cases (16.9\%) & 8 Cases (15.7\%) \\
\hline Pulmonary diseases except infection $\ddagger$ & 7 Cases (4.5\%) & 7 Cases (4\%) & 3 Cases $(5.9 \%)$ \\
\hline Allergy except infusion reaction & 7 Cases (4.5\%) & 12 Cases $(9.2 \%)$ & 6 Cases $(11.8 \%)$ \\
\hline Malignancy & 6 Cases $(3.8 \%)$ & 3 Cases $(2.3 \%)$ & 1 Case $(2 \%)$ \\
\hline Cardiovascular system disease & 2 Cases (1.3\%) & 2 Cases (1.5\%) & 2 Cases $(3.9 \%)$ \\
\hline Good control & 21 Cases $(13.4 \%)$ & 7 Cases (5.4\%) & 2 Cases (3.9\%) \\
\hline Miscellaneous§ & 11 Cases $(7.0 \%)$ & 19 Cases $(14.6 \%) \S$ & 3 Cases $(5.9 \%)$ \\
\hline
\end{tabular}

The $\chi^{2}$ test was applied to assess differences in the proportion of causes for discontinuation (ie, adverse event, lack of efficacy, good control and miscellaneous), and the adjusted residuals were calculated. A significant difference among the three groups $(p=0.026)$ was observed. The adjusted residuals indicated that significantly higher percentages of patients in the infliximab group stopped the treatment due to good disease control compared with the other two groups $(p<0.05)$.

*Values are the number (percentage) of patients who discontinued use because of each reason.

tNumber of patients who discontinued their first biological DMARD for any reason.

$\ddagger$ Pulmonary diseases except for infection included interstitial pneumonia (three cases for infliximab, five for etanercept, two for tocilizumab) and other pulmonary diseases (four for infliximab, two for etanercept, one for tocilizumab).

§Miscellaneous reasons for drug discontinuation include patients' preference, financial reasons, and pregnancy.

DMARD, disease-modifying antirheumatic drugs; $R A$, rheumatoid arthritis.

NA, not applicable

of the observation period was the date these agents were first administered. For patients who started non-biological DMARD at the time of entry in REAL and who later started treatment with biological agents, the start of the observation period was the date of the first administration of biological agents in REAL. Observation was stopped either at 2.5 years after the start of the observation period, on the date of death of a patient, loss to follow up, enrolment in clinical trials, or when therapy was stopped with the first biological agent in REAL for more than 90 days, or on 4 April 2011, whichever came first. The period following switching to a second biological agent was excluded from this study. We defined termination of treatment with biological agents as stopping treatment with the agent for more than 90 days. The date of the last administration of each biological DMARD was retrieved from medical records and reported by the site investigators. Reasons for drug discontinuation were obtained from case report forms of REAL supplemented by medical records, if necessary, and classified into AE, good control, LOE or miscellaneous. We did not discriminate between a primary and secondary LOE. Note that we collected only serious AE in REAL, but also collected AE in this study if it was the main reason for the discontinuation of a biological agent. When a patient had two or more reasons for drug discontinuation, site investigators assigned precedence and we used the primary reason contributing to drug discontinuation for that patient.

\section{Statistical analysis}

The primary outcome of this study was the investigation of the association of the use of infliximab, etanercept and tocilizumab with drug discontinuation due to AE. We also sought to identify other risk factors for drug discontinuation due to AE. Drug retention rates were calculated by the Kaplan-Meier method and compared using the log-rank test among groups. For univariate analysis, the $\chi^{2}$ test was used for comparison of categorical variables and the Kruskal-Wallis test was used for continuous variables among the three agents. For multivariate analysis, the Cox regression hazard model with the forced entry method was employed to compare risks for drug discontinuation due to AE. The validity of the proportional hazards assumption was confirmed by the log-minus-log survival function. We followed the STROBE statement ${ }^{32}$ for clear reporting except for 'the number and reasons for non-participation' in this study.

These statistical analyses were conducted using SPSS (version 16.0Illinois, ). All $p$ values were two-tailed and $p<0.05$ was considered statistically significant.

\section{RESULTS}

\section{Baseline characteristics of the patients}

This analysis included 412 patients in the infliximab group (636.0 patient-years (PY)), 442 in the etanercept group (765.3 PY) and 168 in the tocilizumab group (206.5 PY). Table 1 shows the baseline characteristics of the groups. There were significant differences in age, gender, disease duration and clinical status of the patients. The etanercept and tocilizumab groups had longer disease duration $(p<0.001)$ and higher percentages of comorbidities than the infliximab group ( $p<0.001$ for chronic pulmonary disease, $\mathrm{p}=0.011$ for diabetes mellitus). The rates of biological-naive patients $(96.4 \%$ for the infliximab group, $83.9 \%$ for the etanercept group and $46.4 \%$ for the tocilizumab group) $(\mathrm{p}<0.001)$ and of the use of three or more non-biological DMARD $(p<0.001)$ in the tocilizumab group were the lowest among the three groups. The rate of the use $(\mathrm{p}=0.007)$ and dose $(p<0.001)$ of oral corticosteroids of the etanercept group were higher than those for the other two groups. Disease activity did not differ significantly among the groups.

\section{Occurrence of treatment termination}

The median IOR of the observation period for each group was $1.50(0.74-2.50)$ years for the infliximab group, $2.1(0.98-2.50)$ years for the etanercept group and $1.0(0.5-2.0)$ years for the tocilizumab group. The number of patients who discontinued biological agents for any reason during the observation period was $157(38.1 \%)$ for the infliximab group, $130(29.4 \%)$ for the etanercept group and $51(30.4 \%)$ for the tocilizumab group $\left(\mathrm{p}=0.019\right.$ by $\left.\chi^{2}\right)$. Table 2 shows the reasons for drug discontinuation for each group. A significant difference among the three groups ( $p=0.026$ by $\chi^{2}$ ) was seen in the proportions of reasons for discontinuation, and the adjusted residuals indicated that significantly higher percentages of patients in the infliximab group stopped treatment due to good disease control compared with the other two groups $(p<0.05)$. The most frequently reported 
(A) Adverse events

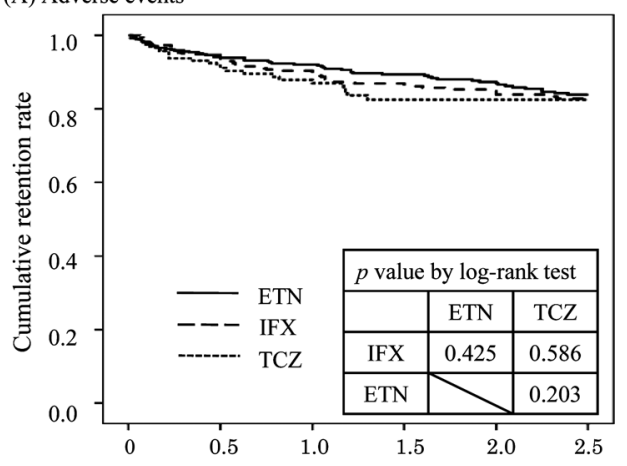

(C) Good control

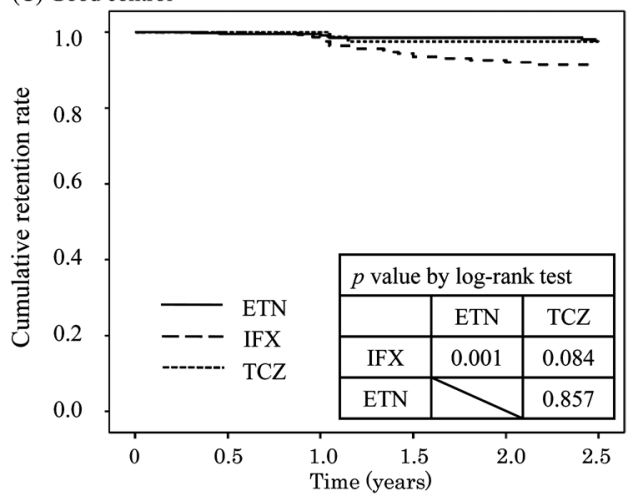

(B) Lack of efficacy

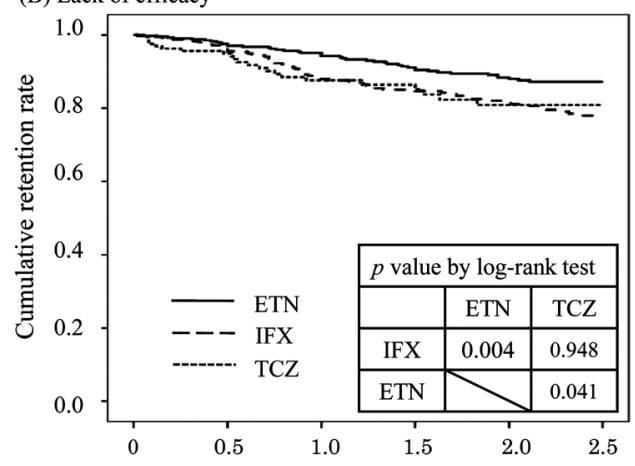

(D) Adverse events and lack of efficacy

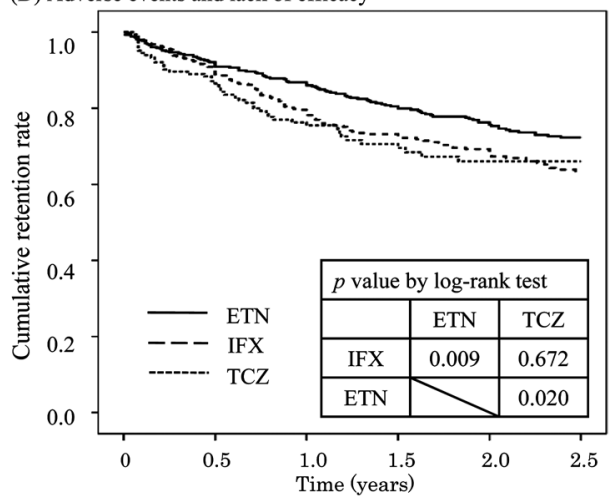

Figure 1 Kaplan-Meier curves for time to discontinuation for each biological agent (etanercept (ETN); infliximab (IFX); tocilizumab (TCZ)). Withdrawal for adverse events (A), lack of efficacy (B), good control (C), and adverse events and lack of efficacy (D) are presented separately. Drug retention rates are compared using the long-rank test among groups. The y axis shows the cumulative retention rates.

reason for discontinuation was LOE in the infliximab group, development of $\mathrm{AE}$ in the etanercept group and both in the tocilizumab group (table 2).

\section{The retention rates of biological agents}

Because the distribution of reasons for drug discontinuation was significantly different among these biological agents (table 2), we investigated drug retention rates per reason for discontinuation. Kaplan-Meier curves for time to discontinuation for each agent due to $\mathrm{AE}$ and $\mathrm{LOE}$ are shown in figure $1 \mathrm{~A}, \mathrm{~B}$, respectively. No significant differences existed among the three drugs for treatment discontinuation due to $\mathrm{AE}$. The discontinuation rate due to LOE was significantly lower for etanercept compared with that of infliximab ( $p=0.004$, log-rank test $)$ and tocilizumab $(p=0.041)$ (figure 1B), and the discontinuation rate for infliximab due to good control was significantly higher than that for etanercept ( $p=0.001$, log-rank test) (figure 1C). We combined withdrawals due to $\mathrm{AE}$ and LOE to assess treatment failure; etanercept had a significantly lower discontinuation rate due to treatment failure compared with the other two agents ( $p=0.009$ vs infliximab, $p=0.020$ vs tocilizumab, log-rank test) (figure $1 D$ ). To evaluate the possible effects of previous treatment with biological agents on drug discontinuation due to $\mathrm{AE}$ and $\mathrm{LOE}$, we compared the retention rates per reason except for good control in the etanercept and tocilizumab groups between biological-naive and non-naive patients (see supplementary figures, available online only). In both groups, there was no significant difference in drug retention rates between biological-naive and non-naive patients. However, we found a numerically higher discontinuation rate of biological agent non-naive patients due to LOE in the tocilizumab group (see supplementary figure S3, available online only).

\section{Multivariate analysis of the risk for discontinuation of biological agents due to $\mathrm{AE}$}

We compared patients who discontinued treatment with biological agents due to $\mathrm{AE}$ and remaining patients using a univariate analysis (see supplementary table S1, available online only) and used the same variables for the multivariate analysis of table 3 . Although we found no significant difference in the use of infliximab and tocilizumab in the univariate analysis (table S1, available online only), the Cox regression hazard model revealed that the adjusted risk for discontinuation due to $\mathrm{AE}$ was significantly higher in patients using infliximab (HR 1.69; 95\% CI 1.14 to 2.51) and tocilizumab (HR 1.98; 95\% CI 1.04 to 3.76 ) compared with etanercept (table 3). Among the other variables, the risk of discontinuation due to AE was also significantly higher in patients with increasing age by decade (HR 1.64; $95 \%$ CI 1.38 to 1.97) and with the previous use of three or more non-biological DMARD (HR 1.86; 95\% CI 1.30 to 2.67).

\section{DISCUSSION}

To our knowledge, this is the first report comparing drug retention rates among TNF inhibitors and tocilizumab and identifying risk factors causing drug discontinuation due to AE. The major findings of this study are: (1) the reasons for discontinuation were significantly different among the three biological agents studied; (2) the risk of discontinuation due to $\mathrm{AE}$ was significantly higher in patients using infliximab and tocilizumab compared with etanercept; and (3) other significant risk factors for the discontinuation due to $\mathrm{AE}$ were increasing age and the previous use of three or more non-biological DMARD.

There are some reports describing drug retention rates and reasons for drug discontinuations in patients treated with TNF 
Table 3 Multivariate analysis for drug discontinuation due to adverse events in RA patients treated with infliximab, etanercept or tocilizumab*

\begin{tabular}{llr}
\hline & HR (95\% Cl) & p Value \\
\hline Infliximab (vs etanercept) & 1.69 (1.14 to 2.51) & 0.009 \\
Tocilizumab (vs etanercept) & 1.98 (1.04 to 3.76) & 0.037 \\
Age by decade & 1.64 (1.38 to 1.97) & $<0.001$ \\
Class 3 or 4 (vs class 1 or 2) & 1.07 (0.74 to 1.54) & 0.727 \\
DAS28 (3/CRP) at baseline (per 1.0 increment) & 1.03 (0.92 to 1.17) & 0.585 \\
Chronic pulmonary disease & 1.19 (0.83 to 1.70) & 0.336 \\
Diabetes mellitus & 0.95 (0.58 to 1.56) & 0.841 \\
Concomitant use of oral corticosteroids at baseline & $1.15(0.78$ to 1.70$)$ & 0.489 \\
Concomitant use of immunosuppressive drugs except for methotrexate at baseline & $0.56(0.20$ to 1.55$)$ & 0.262 \\
Previous use of three or more non-biological DMARD & 1.86 (1.30 to 2.67) & 0.001 \\
Previous use of biological agents & 1.05 (0.64 to 1.72) & 0.842 \\
\hline
\end{tabular}

${ }^{*}$ Cox regression hazard model analysis, adjusted for the variables included in the table, gender and calendar year.

Class, Steinbrocker's class; CRP, C-reactive protein;

DAS28, disease activity score including 28-joint count; DMARD, disease-modifying antirheumatic drug; RA, rheumatoid arthritis.

inhibitors. ${ }^{14}$ 16-18 2022242627 33-35 Among patients stopping treatment with TNF inhibitors due to any reason, approximately half of those discontinued due to $\mathrm{AE}$, and the proportions of patients who discontinued the agents due to AE or LOE were similar in each group in the Swiss ${ }^{14}$ and the French ${ }^{16}$ registries. In this study, AE and LOE were the two major reported reasons for discontinuation, with similar percentages also for all three groups, but the discontinuation rate due to good control in the infliximab group was significantly higher than those in the other two groups. Several studies have shown successful discontinuation of treatment with infliximab ${ }^{10} 6^{30}$ and tocilizumab 41 without flare of RA, but the reported percentage of patients who could discontinue infliximab was higher compared with tocilizumab. In contrast, there is no evidence of the successful discontinuation of treatment for etanercept to date. Therefore, our results might be influenced by physicians' expectations for successful discontinuation of biological agents based on previous reports.

We observed a significantly lower discontinuation rate due to LOE in the etanercept group compared with infliximab and tocilizumab (figure.1B), which can be explained by the following reasons. First, treatment with infliximab induces the formation of human antichimeric antibody in some patients, which may lead to LOE or adverse drug reactions. ${ }^{42} 43$ The prevalence of antidrug antibodies in RA patients who were treated with infliximab is much higher compared with etanercept ${ }^{44} 45$ and tocilizumab. ${ }^{15} 46$ Second, the tocilizumab group had a significantly lower percentage of biological-naive patients, which may be associated with a less favourable response to treatment. ${ }^{47} 48$ In the tocilizumab group, we confirmed that the discontinuation rate due to LOE was numerically lower in the biological-naive patients compared with biological agent non-naive patients (see supplementary figure S3, available online only).

In this study, we limited our multivariate analyses to the risk factors associated with discontinuation due to AE. Some previous studies identified risk factors for overall discontinuation in patients treated with TNF inhibitors. ${ }^{6} 1726$ Because treatments with biological agents are discontinued for various reasons, as shown in table 1, we postulated that it would not be appropriate to build a multivariate model for overall discontinuation from a medical point of view. In REAL, we did not collect measures of patients' disease activity, such as the disease activity score in 28 joints (DAS28), when patients stopped treatment with biological agents, and we could not define discontinuation due to LOE by using objective criteria. Therefore, we opted not to analyse risk factors for discontinuation due to LOE. The number of patients who discontinued the agents due to good control was too small to analyse associated factors using multivariate analysis.

Increasing age was also identified as a risk factor associated with the discontinuation of biological agents due to AE, data supported by a previous report. ${ }^{16}$ In all three groups, infections were most frequent among AE leading to drug discontinuation (table 2). It is plausible that increasing age contributes to discontinuation because of an increasing risk of RA patients for infection 2949 with age. Higher numbers of previous nonbiological DMARD use suggests cases difficult to treat, with high disease activity or long-standing disease. Compatible with this possibility, patients who had been treated with three or more non-biological DMARD before enrolment in REAL had a significantly longer disease duration with more advanced disease stages and classes than those receiving less than three nonbiological DMARD (data not shown). It has been reported that advanced stage or higher disease activity was reported as a risk for infections. 82950

Our study has limitations. First, we have to mention the possibility of selection bias in this study. However, because almost all patients who were registered from the participating hospitals of our study to the all-cases postmarketing surveillance programmes for each biological DMARD were enrolled in REAL, selection bias was substantially decreased. Second, we analysed the first biological agent administered to each patient at or after enrolment in REAL. However, these biological agents were not necessarily truly the first one used for each patient; rates of biological-naive patients were significantly different among the three groups (table 1), indicating the presence of channelling bias. Therefore, we adjusted for the previous use of biological agents in the multivariate analysis.

In conclusion, we have presented the first epidemiological data that directly compare TNF inhibitors and tocilizumab in a single cohort. We demonstrated that reasons for discontinuation were significantly different among the biological agents and that the use of infliximab and tocilizumab had a significantly higher risk of treatment discontinuation due to AE compared with etanercept after adjusting for various confounding factors.

Values are the mean \pm SD, unless otherwise stated. For univariate analysis, the $\chi^{2}$ test for categorical variables and the Student's t test or Mann-Whitney test were used to compare continuous variables among groups.

Steinbrocker's classification ${ }^{30}$ was used to definite RA disease stages and classes.

The immunosuppressive drugs used were tacrolimus, leflunomide, mizoribine and ciclosporin. 
The oral corticosteroid dose was converted to the equivalent prednisolone dosage. Methotrexate and corticosteroid doses are shown as the mean $\pm \mathrm{SD}$ among users of these drugs.

Chronic pulmonary diseases include interstitial pneumonia, chronic obstructive pulmonary disease, bronchial asthma, previous pulmonary tuberculosis and bronchiectasis.

Acknowledgements The investigators of the REAL study group and their affiliates who contributed to this work were: Yukiko Komano (Tokyo Medical and Dental University); Shintaro Hirata (University of Occupational and Environmental Health); Taichi Hayashi (University of Tsukuba); Mitsuhiro Takeno (Yokohama City University); Shinsuke Yasuda (Hokkaido University); Yoshinari Takanasaki (Juntendo University); Tsuneyo Mimori (Kyoto University); Syunichi Shiozawa (Kobe University); Hiroaki Ida, Katsumi Eguchi (Nagasaki University); Kazuhiko Yamamoto (The university of Tokyo): Kazuhiko Ezawa (Kurashiki Kohsai Hospital); Sae Ochi (Tokyo Metropolitan Bokutoh Hospital); Kenji Nagasaka (Ome Municipal General Hospital); Hideto Kameda, Yuko Kaneko, Tsutomu Takeuchi (Keio University); Kiyoshi Migita (National Hospital Organization Nagasaki Medical Centre); Yasushi Miura (Kobe University); Tetsuji Sawada (Tokyo Medical University Hospital): Ayako Nakajima, Hisashi Yamanaka (Tokyo Women's Medical University); Yoshinori Nonomura (Tokyo Kyosai Hospital). Yokohama City Minato Red Cross Hospital is also a member of the REAL study group, but was not involved in the present study. The authors sincerely thank all the rheumatologists and others caring for RA patients enrolled in REAL.

Funding This work was supported by a grant-in-aid from the Ministry of Health, Labour and Welfare, Japan (H23-meneki-sitei-016 and H19-meneki-ippan-009 to NM, H22-meneki-ippann-001 to $\mathrm{MH}$ ) and by a grant-in-aid for scientific research from the Japan Society for the Promotion of Science (\#20390158 to MH, \#19590530 to RK, and \#50277141 to MT). This work was also supported by grants for pharmacovigilance research on biological agents from Abbott Laboratories, Bristol-Myers Japan, Eisai, Chugai Pharmaceutical, Mitsubishi Tanabe Pharma Corp, Takeda Pharmaceutical and Pfizer Japan (to MH), and by a grant from the Japanese Ministry of Education, Global Center of Excellence (GCOE) Program, 'International Research Center for Molecular Science in Tooth and Bone Diseases'.

Competing interests KA has received research support from Chugai Pharmaceutical, Mitsubishi Tanabe Pharma and Astellas Pharma. YT has received consulting fees, speaking fees, and/or honoraria from Mitsubishi-Tanabe Pharma, Chugai Pharmaceutical, Eisai, Takeda Pharmaceutical, Astellas Pharma and Abbott Japan, and has received research grant support from Mitsubishi-Tanabe Pharma, Takeda Pharmaceutical, MSD KK, Pfizer Japan, Astellas Pharma, Chugai Pharmaceutical, Abbott Japan and Eisai. TF has received grant/research support from Abbott Japan, Eisai, Takeda Pharmaceutical, Mitsubishi Tanabe Pharma, Chugai Pharmaceutical, Pfizer Japan, Astellas Pharma, Bristol-Myers Squibb KK. NM has received research grants from Abbott Japan, Astellas Pharma, MSD KK, Chugai Pharmaceutical, Daiichi Sankyo, Eisai, Janssen Pharmaceutical KK, Mitsubishi Tanabe Pharma, Takeda Pharmaceutical and Teijin Pharma. MH has received research grants from Abbott Japan, Astellas Pharma, Bristol Myers Squibb KK, Chugai Pharmaceutical, Eisai, Janssen Pharmaceutical KK, Mitsubishi Tanabe Pharma, Santen Pharmaceutical, Takeda Pharmaceutical and Pfizer Japan.

Ethics approval The REAL study was approved by the ethics committees of the Tokyo Medical and Dental University Hospital and other participating institutions.

\section{Patient consent Obtained}

Provenance and peer review Not commissioned; externally peer reviewed.

Author affiliations 'Department of Pharmacovigilance, Graduate School of Medical and Dental Sciences, Tokyo Medical and Dental University, Tokyo, Japan ${ }^{2}$ Department of Medicine and Rheumatology, Graduate School of Medical and Dental Sciences, Tokyo Medical and Dental University, Tokyo, Japan

${ }^{3}$ Clinical Research Center, Tokyo Medical and Dental University Hospital, Tokyo, Japan

${ }^{4}$ Department of Rheumatology/Clinical Immunology, Saitama Medical Center, Saitama Medical University, Kawagoe, Japan

${ }^{5}$ The First Department of Internal Medicine, University of Occupational and Environmental Health, Japan, Kitakyushu, Japan

${ }^{6}$ Division of Clinical Immunology, Doctoral Program in Clinical Sciences, Graduate School of Comprehensive Human Sciences, University of Tsukuba, Tsukuba, Japan ${ }^{7}$ Department of Internal Medicine and Clinical Immunology, Yokohama City University Graduate School of Medicine, Yokohama, Japan

${ }^{8}$ Department of Internal Medicine II, Hokkaido University, Graduate School of Medicine, Sapporo, Japan

${ }^{9}$ Department of Rheumatology, Tokyo Metropolitan Police Hospital, Tokyo, Japan

${ }^{10}$ Department of Internal Medicine and Rheumatology, Juntendo University School of Medicine, Tokyo, Japan

${ }^{11}$ Department of the Control for Rheumatic Diseases, Graduate School of Medicine, Kyoto University, Kyoto, Japan
12Department of Internal Medicine, Division of Endocrinology and Metabolism, Hematology, Rheumatology and Respiratory Medicine, Faculty of Medicine, Kagawa University, Kagawa, Japan

${ }^{13}$ Department of Rheumatology, Clinical Research Center for Allergy and Rheumatology, Sagamihara National Hospital, National Hospital Organization,

Sagamihara, Japan

${ }^{14}$ Tokyo Metropolitan Geriatric Hospital, Tokyo, Japan

${ }^{15}$ Rheumatic and Collagen Disease Center, Sasebo Chuo Hospital, Nagasaki, Japan

${ }^{16}$ Department of Rheumatology, Kobe University Graduate School of Medicine, Kobe, Japan

${ }^{17}$ Unit of Translational Medicine, Department of Immunology and Rheumatology, Nagasaki University Graduate School of Biomedical Sciences, Nagasaki University, Nagasaki, Japan

${ }^{18}$ Department of Allergy and Rheumatology, The University of Tokyo, Tokyo, Japan ${ }^{19} \mathrm{Global}$ Center of Excellence (GCOE) Program; International Research Center for Molecular Science in Tooth and Bone Diseases, Tokyo Medical and Dental University, Tokyo, Japan

\section{REFERENCES}

1. Saag KG, Teng GG, Patkar NM, et al. American College of Rheumatology 2008 recommendations for the use of nonbiologic and biologic disease-modifying antirheumatic drugs in rheumatoid arthritis. Arthritis Rheum 2008;59:762-84.

2. Knevel R, Schoels M, Huizinga TW, et al. Current evidence for a strategic approach to the management of rheumatoid arthritis with disease-modifying antirheumatic drugs: a systematic literature review informing the EULAR recommendations for the management of rheumatoid arthritis. Ann Rheum Dis 2010;69:987-94.

3. Koike R, Takeuchi T, Eguchi K, et al. Update on the Japanese guidelines for the use of infliximab and etanercept in rheumatoid arthritis. Mod Rheumatol 2007;17:451-8.

4. Koike R, Harigai M, Atsumi T, et al. Japan College of Rheumatology 2009 guidelines for the use of tocilizumab, a humanized anti-interleukin-6 receptor monoclonal antibody, in rheumatoid arthritis. Mod Rheumatol 2009;19:351-7.

5. Koike T, Harigai M, Inokuma $S$, et al. Postmarketing surveillance of the safety and effectiveness of etanercept in Japan. J Rheumatol 2009;36:898-906.

6. Tanaka Y, Takeuchi T, Inoue E, et al. Retrospective clinical study on the notable efficacy and related factors of infliximab therapy in a rheumatoid arthritis management group in Japan: one-year clinical outcomes (RECONFIRM-2). Mod Rheumatol 2008;18:146-52.

7. Yamanaka $\mathbf{H}$, Tanaka $Y$, Inoue $E$, et al. Efficacy and tolerability of tocilizumab in rheumatoid arthritis patients seen in daily clinical practice in Japan: results from a retrospective study (REACTION study). Mod Rheumatol 2011;21:122-33.

8. Takeuchi T, Tatsuki Y, Nogami Y, et al. Postmarketing surveillance of the safety profile of infliximab in 5000 Japanese patients with rheumatoid arthritis. Ann Rheum Dis 2008;67:189-94.

9. Smolen JS, Landewé R, Breedveld FC, et al. EULAR recommendations for the management of rheumatoid arthritis with synthetic and biological disease-modifying antirheumatic drugs. Ann Rheum Dis 2010;69:964-75.

10. Tanaka Y, Takeuchi T, Mimori T, et al. Discontinuation of infliximab after attaining low disease activity in patients with rheumatoid arthritis: RRR (remission induction by Remicade in RA) study. Ann Rheum Dis 2010;69:1286-91.

11. Klarenbeek NB, van der Kooij SM, Güler-Yüksel M, et al. Discontinuing treatment in patients with rheumatoid arthritis in sustained clinical remission: exploratory analyses from the BeSt study. Ann Rheum Dis 2011;70:315-9.

12. Harigai $\mathbf{M}$, Takeuchi $T$, Tanaka $Y$, et al. Discontinuation of adalimumab treatment in rheumatoid arthritis patients after achieving low disease activity. Mod Rheumatol Published Online First: 20 January 2012. doi:10.1007/s10165-011-0586-5.

13. Vander Cruyssen B, Durez P, Westhovens R, et al. Seven-year follow-up of infliximab therapy in rheumatoid arthritis patients with severe long-standing refractory disease: attrition rate and evolution of disease activity. Arthritis Res Ther 2010;12:R77

14. Du Pan SM, Dehler S, Ciurea A, et al. Comparison of drug retention rates and causes of drug discontinuation between anti-tumor necrosis factor agents in rheumatoid arthritis. Arthritis Rheum 2009;61:560-8.

15. Nishimoto N, Miyasaka N, Yamamoto K, et al. Long-term safety and efficacy of tocilizumab, an anti-IL-6 receptor monoclonal antibody, in monotherapy, in patients with rheumatoid arthritis (the STREAM study): evidence of safety and efficacy in a 5-year extension study. Ann Rheum Dis 2009;68:1580-4.

16. Marchesoni A, Zaccara E, Gorla R, et al. TNF-alpha antagonist survival rate in a cohort of rheumatoid arthritis patients observed under conditions of standard clinical practice. Ann NY Acad Sci 2009;1173:837-46.

17. Hetland ML, Christensen IJ, Tarp U, et al. Direct comparison of treatment responses, remission rates, and drug adherence in patients with rheumatoid arthritis treated with adalimumab, etanercept, or infliximab: results from eight years of surveillance of clinical practice in the nationwide Danish DANBIO registry. Arthritis Rheum 2010;62:22-32

18. Kristensen LE, Saxne T, Nilsson JA, et al. Impact of concomitant DMARD therapy on adherence to treatment with etanercept and infliximab in rheumatoid arthritis. Results from a six-year observational study in southern Sweden. Arthritis Res Ther 2006;8:R174. 
19. Glinthorg B, Østergaard M, Dreyer L, et al. Treatment response, drug survival, and predictors thereof in 764 patients with psoriatic arthritis treated with anti-tumor necrosis factor a therapy: results from the nationwide Danish DANBIO registry. Arthritis Rheum 2011;63:382-90.

20. Soliman MM, Ashcroft DM, Watson KD, et al. Impact of concomitant use of DMARDs on the persistence with anti-TNF therapies in patients with rheumatoid arthritis: results from the British Society for Rheumatology Biologics Register. Ann Rheum Dis 2011;70:583-9.

21. Wendling D, Materne GE, Michel F, et al. Infliximab continuation rates in patients with rheumatoid arthritis in everyday practice. Joint Bone Spine 2005;72:309-12.

22. Duclos M, Gossec L, Ruyssen-Witrand A, et al. Retention rates of tumor necrosis factor blockers in daily practice in 770 rheumatic patients. $J$ Rheumatol 2006;33:2433-8.

23. Koike T, Harigai M, Inokuma $\mathrm{S}$, et al. Postmarketing surveillance of safety and effectiveness of etanercept in Japanese patients with rheumatoid arthritis. Mod Rheumatol 2011;21:343-51.

24. Kievit W, Fransen J, Adang EM, et al. Long-term effectiveness and safety of TNF-blocking agents in daily clinical practice: results from the Dutch Rheumatoid Arthritis Monitoring register. Rheumatology (Oxford) 2011;50:196-203.

25. Leffers HC, Ostergaard M, Glintborg B, et al. Efficacy of abatacept and tocilizumab in patients with rheumatoid arthritis treated in clinical practice: results from the nationwide Danish DANBIO registry. Ann Rheum Dis 2011;70:1216-22.

26. Zink A, Listing J, Kary S, et al. Treatment continuation in patients receiving biological agents or conventional DMARD therapy. Ann Rheum Dis 2005;64:1274-9.

27. Greenberg JD, Reed G, Decktor D, et al. A comparative effectiveness study of adalimumab, etanercept and infliximab in biologically naive and switched rheumatoid arthritis patients: results from the US CORRONA registry. Ann Rheum Dis Published Online First: 30 January 2012. doi:10.1136/annrheymdis-2011-150573.

28. Sakai R, Komano Y, Tanaka M, et al. The REAL database reveals no significant risk of serious infection during treatment with a methotrexate dose of more than $8 \mathrm{mg} /$ week in patients with rheumatoid arthritis. Mod Rheumatol 2011;21:444-8.

29. Komano $\mathbf{Y}$, Tanaka M, Nanki T, et al. Incidence and risk factors for serious infection in patients with rheumatoid arthritis treated with tumor necrosis factor inhibitors: a report from the Registry of Japanese Rheumatoid Arthritis Patients for Longterm Safety. J Rheumatol 2011;38:1258-64.

30. Steinbrocker $\mathbf{0}$, Traeger $\mathrm{CH}$, Batterman $\mathrm{RC}$. Therapeutic criteria in rheumatoid arthritis. JAMA 1949;140:659-62.

31. Bruce B, Fries JF. The Stanford Health Assessment Questionnaire: dimensions and practical applications. Health Qual Life Outcomes 2003;1:20.

32. von Elm E, Altman DG, Egger $\mathrm{M}$, et al. Strengthening the Reporting of Observational Studies in Epidemiology (STROBE) statement: guidelines for reporting observational studies. BMJ 2007;335:806-8.

33. Yazici Y, Krasnokutsky S, Barnes JP, et al. Changing patterns of tumor necrosis factor inhibitor use in 9074 patients with rheumatoid arthritis. J Rheumatol 2009:36:907-13.

34. Grijalva CG, Chung CP, Arbogast PG, et al. Assessment of adherence to and persistence on disease-modifying antirheumatic drugs (DMARDs) in patients with rheumatoid arthritis. Med Care 2007;45(10 Suppl 2):S66-76.

35. Blom M, Kievit W, Fransen J, et al. The reason for discontinuation of the first tumor necrosis factor (TNF) blocking agent does not influence the effect of a second TNF blocking agent in patients with rheumatoid arthritis. J Rheumatol 2009;36:2171-7.

36. Quinn MA, Conaghan PG, O'Connor PJ, et al. Very early treatment with infliximab in addition to methotrexate in early, poor-prognosis rheumatoid arthritis reduces magnetic resonance imaging evidence of synovitis and damage, with sustained benefit after infliximab withdrawal: results from a twelve-month randomized, double-blind, placebo-controlled trial. Arthritis Rheum 2005;52:27-35.

37. Goekoop-Ruiterman YP, de Vries-Bouwstra JK, Allaart CF, et al. Comparison of treatment strategies in early rheumatoid arthritis: a randomized trial. Ann Intern Med 2007:146:406-15.

38. Goekoop-Ruiterman YP, de Vries-Bouwstra JK, Allaart CF, et al. Clinical and radiographic outcomes of four different treatment strategies in patients with early rheumatoid arthritis (the BeSt study): a randomized, controlled trial. Arthritis Rheum 2008;58 (2 Suppl):S126-35.

39. van der Bijl AE, Goekoop-Ruiterman YP, de Vries-Bouwstra JK, et al. Infliximab and methotrexate as induction therapy in patients with early rheumatoid arthritis. Arthritis Rheum 2007:56:2129-34.

40. van der Kooij SM, Goekoop-Ruiterman YP, de Vries-Bouwstra JK, et al. Drug-free remission, functioning and radiographic damage after 4 years of response-driven treatment in patients with recent-onset rheumatoid arthritis. Ann Rheum Dis 2009;68:914-21.

41. Nishimoto $\mathbf{N}$. Drug Free Remission After Cessation Of Tocilizumab (Actemra) Monotherapy (DREAM Study). Arthritis Rheum 2010;62:S750.

42. Maini RN, Breedveld FC, Kalden JR, et al. Therapeutic efficacy of multiple intravenous infusions of anti-tumor necrosis factor alpha monoclonal antibody combined with low-dose weekly methotrexate in rheumatoid arthritis. Arthritis Rheum 1998:41:1552-63

43. Wolbink GJ, Vis M, Lems W, et al. Development of antiinfliximab antibodies and relationship to clinical response in patients with rheumatoid arthritis. Arthritis Rheum 2006;54:711-15.

44. Emi Aikawa N, de Carvalho JF, Artur Almeida Silva C, et al. Immunogenicity of AntiTNF-alpha agents in autoimmune diseases. Clin Rev Allergy Immunol 2010;38:82-9.

45. Taylor PC. Pharmacology of TNF blockade in rheumatoid arthritis and other chronic inflammatory diseases. Curr Opin Pharmacol 2010;10:308-15.

46. Maini RN, Taylor PC, Szechinski J, et al. Double-blind randomized controlled clinical trial of the interleukin-6 receptor antagonist, tocilizumab, in European patients with rheumatoid arthritis who had an incomplete response to methotrexate. Arthritis Rheum 2006;54:2817-29.

47. Nakashima $\mathbf{Y}$, Kondo $\mathbf{M}$, Harada $\mathrm{H}$, et al. Clinical evaluation of tocilizumab for patients with active rheumatoid arthritis refractory to anti-TNF biologics: tocilizumab in combination with methotrexate. Mod Rheumatol 2010;20:343-52.

48. Burmester GR, Feist $\mathrm{E}$, Kellner $\mathrm{H}$, et al. Effectiveness and safety of the interleukin 6-receptor antagonist tocilizumab after 4 and 24 weeks in patients with active rheumatoid arthritis: the first phase Illb real-life study (TAMARA). Ann Rheum Dis 2011;70:755-9.

49. Favalli EG, Desiati F, Atzeni F, et al. Serious infections during anti-TNFalpha treatment in rheumatoid arthritis patients. Autoimmun Rev 2009;8:266-73.

50. Au K, Reed G, Curtis JR, et al. High disease activity is associated with an increased risk of infection in patients with rheumatoid arthritis. Ann Rheum Dis 2011;70:785-91. 


\section{Drug retention rates and relevant risk factors for drug discontinuation due to adverse events in rheumatoid arthritis patients receiving anticytokine therapy with different target molecules}

Ryoko Sakai, Michi Tanaka, Toshihiro Nanki, et al.

Ann Rheum Dis 2012 71: 1820-1826 originally published online April 13,2012

doi: 10.1136/annrheumdis-2011-200838

Updated information and services can be found at:

http://ard.bmj.com/content/71/11/1820.full.html

\section{These include:}

\section{Data Supplement}

References

Email alerting
service
"Web Only Data"

http://ard.bmj.com/content/suppl/2012/04/12/annrheumdis-2011-200838.DC1.html

This article cites 48 articles, 19 of which can be accessed free at: http://ard.bmj.com/content/71/11/1820.full.html\#ref-list-1

Article cited in:

http://ard.bmj.com/content/71/11/1820.full.html\#related-urls

Receive free email alerts when new articles cite this article. Sign up in the box at the top right corner of the online article.

\section{Topic \\ Collections}

Articles on similar topics can be found in the following collections

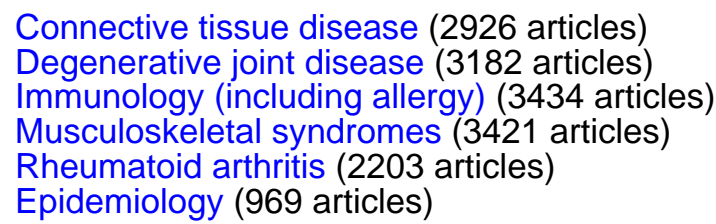

To request permissions go to:

http://group.bmj.com/group/rights-licensing/permissions

To order reprints go to:

http://journals.bmj.com/cgi/reprintform

To subscribe to BMJ go to:

http://group.bmj.com/subscribe/ 


\section{Notes}

To request permissions go to:

http://group.bmj.com/group/rights-licensing/permissions

To order reprints go to:

http://journals.bmj.com/cgi/reprintform

To subscribe to BMJ go to:

http://group.bmj.com/subscribe/ 